\title{
Clinical Characteristics of Patients and Factors Associated with Switching Biologics in Asthma
}

\author{
Machiko Matsumoto-Sasaki ', Kaoruko Simizu', Masanobu Suzuki ${ }^{2}$, Masaru Suzuki ', Hirokazu Kimura', \\ Yuji Nakamaru², Yoichi M Ito ${ }^{3}$, Akihiro Honma ${ }^{2}$, Satoshi Konno' \\ 'Department of Respiratory Medicine, Faculty of Medicine and Graduate School of Medicine, Hokkaido University, Sapporo, Japan; ${ }^{2}$ Department of \\ Otolaryngology-Head and Neck Surgery, Faculty of Medicine and Graduate School of Medicine, Hokkaido University, Sapporo, Japan; ${ }^{3}$ Data Science \\ Center, Promotion Unit, Institute of Health Science Innovation for Medical Care, Hokkaido University Hospital, Sapporo, Japan
}

Correspondence: Kaoruko Simizu, Department of Respiratory Medicine, Faculty of Medicine and Graduate School of Medicine, Hokkaido University, Kita 15, Nishi 7, Kita-ku, Sapporo, Japan, Tel +8I-I I-706-59I I, Fax +8I-I I-706-7899, Email okaoru@med.hokudai.ac.jp

Purpose: Biologics have been used increasingly for the treatment of severe asthma. However, established guidelines for the selection, switching, or discontinuation of biologics do not exist. We aimed to identify the clinical characteristics of patients with asthma who required switching biologics and the factors associated with switching biologics.

Patients and Methods: This was a retrospective study of 42 patients with severe asthma treated with biologics at the Hokkaido University Hospital between $23^{\text {rd }}$ June 2016 and $30^{\text {th }}$ April 2021, when two biologics were available in Japan. We compared the characteristics of subjects who continued and switched biologics. The time to switch the biologics was assessed by type 2 inflammatory biomarkers, pulmonary function indices, and the presence of comorbidities, including the Japanese Epidemiological Survey of Refractory Eosinophilic Chronic Rhinosinusitis (JESREC) score and aspirin exacerbated respiratory diseases (AERD), using the Kaplan-Meier method and a multivariate Cox proportional hazards model.

Results: Eight and five patients were treated by mepolizumab and benralizumab at baseline, respectively among the $31 \%$ (13/42) who switched the biologics. Subjects who required switching biologics were characterized by high blood eosinophil counts, younger age, JESREC scores of 11 points or higher, and AERD. The time taken to switch biologics was significantly shorter in the subgroups with high JESREC scores $(\geq 11)$ or AERD, compared with their counterparts with low JESREC scores or without AERD (both, P $<0.05$ ). JESREC scores of $\geq 11$, but not the presence of AERD, were associated with time to switch biologics.

Conclusion: The presence of eosinophilic chronic rhinosinusitis based on JESREC scores of $\geq 11$ and younger age were factors associated with switching biologics in asthma.

Keywords: aspirin-exacerbated respiratory disease, asthma, biologics, comorbidities, eosinophilic chronic rhinosinusitis

\section{Introduction}

Biologic therapies have played a significant role in the treatment of severe asthma. " 1 ifficult-to-treat asthma is asthma that is uncontrolled, despite receiving medium or high dose Inhaled corticosteroids (ICS)-long-acting beta2agonist (LABA) treatment or requires high dose ICS-LABA treatment to maintain adequate symptom control and reduce exacerbations, which incorrect inhalation technique, poor adherence to the medication, smoking, comorbidities, or misdiagnosis may concern. Severe asthma is a subset of difficult-to-treat asthma that is uncontrolled despite adherence to maximal optimal therapy and treatment of contributory factors or worsens when the dosage of the medication is decreased. ${ }^{2,3}$ Poor asthma control is defined as an Asthma Control Test (ACT) score of 19 or less. ${ }^{4}$ Symptom control, frequency of exacerbations, and pulmonary function should be evaluated in the course of treatment including biologics. ${ }^{2}$

In Japan, four types of biologics are currently in use. ${ }^{5}$ There are no established guidelines for the use of different biologics, ${ }^{2,5-7}$ although some reports suggest that type 2 markers such as blood eosinophil counts, fractional exhaled nitric oxide (FeNO), and IgE could act as eligibility criteria for selecting biologic therapy. Practically, there is substantial 
overlap in the proposed indications for biologics, which leaves the ultimate decision at the discretion of the treating clinician. Thus, some patients have to discontinue, which requires amelioration to achieve optimal treatment.

The definitions of responders have been applied to evaluate the efficacy of biologics. ${ }^{8}$ However, these definitions have not been validated. Eger et al defined patients who achieved $\geq 50 \%$ reduction in exacerbations as responders and $\geq 50 \%$ reduction in prednisolone dose as a response to biologics. ${ }^{9}$ Non-responders are defined as patients who discontinued antiinterleukin IL-5 (anti-IL-5) treatment following less than 2 years of treatment because of clinical worsening and increased symptoms, decreased forced expiratory volume in 1 second $\left(\mathrm{FEV}_{1}\right)$, or increased oral corticosteroid (OCS) use. ${ }^{10}$ The significance of clinical studies in the real-world management of asthma using biologics can be augmented by applying a standard definition of responders and non-responders. Notably, examining the features of non-responders would facilitate the exploration of novel biomarkers or approaches for the management of severe asthma.

Severe asthma is often accompanied by comorbidities such as eosinophilic chronic rhinosinusitis (ECRS), allergic rhinitis (AR), gastroesophageal reflux disease (GERD), and atopic dermatitis (AD). In addition to poor control of asthma which is the main reason for the switching, comorbidities such as sinusitis are reported to be a reason for switching from the treatment of anti-IL-5 biologics (mepolizumab, reslizumab, benralizumab). ${ }^{10}$ Although these comorbidities may affect the clinical course of asthma and may interact pathophysiologically, most clinical studies evaluate individual organ responses to biologics, as opposed to the combined responses of multiple organs. We have reported that a differential response to biologics can be experienced in asthma and ECRS. ${ }^{11,12}$ Thus, it may be plausible that multidimensional evaluations should be considered to provide personalized treatment to asthmatic patients.

The present study aimed to identify the clinical characteristics of asthmatics requiring switching among biologic therapies and factors associated with switching biologics. We first compared the characteristics, including comorbidities, between those who required switching biologics and those treated successfully with first-line biologics. We also examined the clinical factors associated with the time to switching biologics.

\section{Methods}

\section{Study Population}

This study was conducted in accordance with the Declaration of Helsinki. The study was approved by Ethical Review Board for Life Science and Medical Research, Hokkaido University Hospital (approval number: 021-0080). Informed consent was obtained in the form of an opt-out option on the website. We retrospectively reviewed the medical records of patients with severe asthma treated with biologics at the Hokkaido University Hospital between $1^{\text {st }}$ March 2009, when omalizumab was introduced, and $30^{\text {th }}$ April 2021. The analysis period lasted from $23^{\text {rd }}$ June 2016, when mepolizumab became available in Japan, to the $30^{\text {th }}$ April 2021. Since this study focused on switching biologics, we excluded patients who discontinued omalizumab before the introduction of an alternative biologic (mepolizumab) on $23^{\text {rd }}$ June 2016. The observed time to switch biologics started on 1) $23^{\text {rd }}$ June 2016 in case of patients in whom omalizumab was administered before $23^{\text {rd }}$ June 2016, and 2) the date of starting a biologic in case of patients in whom a biologic was administered on or after $23^{\text {rd }}$ June 2016. The diagnosis of severe asthma was based on the ERS/ATS guidelines for adult asthma. ${ }^{3}$ Patients were divided into two groups: those who were treated successfully with first-line biologic therapy during the observational period (continuous group) and those who required switching the initial biologic drug (switched group).

Data regarding patient characteristics, blood eosinophil counts, and serum total $\operatorname{IgE}$ were collected from the clinical documents. The blood eosinophil counts, serum total IgE, pulmonary function tests, FeNO, and comorbidity data were collected before the observed biologic was introduced to allow for the baseline analysis. The presence of atopy was defined as a positive specific IgE test result for at least one common inhaled allergen.

\section{FeNO Measurement and Pulmonary Function Tests at Baseline}

FeNO concentration was measured with the NIOX MINO ${ }^{\circledR}$ (Aerocrine, Stockholm, Sweden) using a single-breath online method, following the ATS guideline. ${ }^{13}$ Spirometry, including FEV $_{1}$ and forced vital capacity (FVC) was performed using a rolling seal Chestac-33 spirometer (Chest MI, Tokyo, Japan), according to the Japanese Respiratory Society guidelines. $^{14}$ 


\section{Comorbidities at Baseline}

We defined ECRS as a Japanese Epidemiological Survey of Refractory Eosinophilic Chronic Rhinosinusitis (JESREC) score of 11 or higher. ${ }^{15}$ The JESREC score is an objective clinical assessment for the diagnosis of ECRS ${ }^{15}$ and consists of bilateral disease sites, nasal polyps, computed tomography (CT) findings, and eosinophilia in the peripheral blood. Aspirin-exacerbated respiratory disease (AERD) is characterized by asthma, intolerance to aspirin and other NSAIDs, chronic rhinosinusitis, mast cell activation, and the overproduction of cysteinyl leukotrienes. ${ }^{16}$ The definition of AERD is based on the history of worsening of symptoms with NSAIDs including aspirin, the judgment of the attending physician, and medical records. Eosinophilic otitis media (EOM) is strongly associated with asthma, and chronic rhinosinusitis is strongly associated with nasal polyps (CRSwNP). ${ }^{17}$ EOM was defined as refractory otitis media, diagnosed by an otolaryngologist. We classified subjects as having AR if they responded affirmatively to the question, "Do you have any nasal allergies including hay fever?" AR was diagnosed by the attending physician or otolaryngologist.

\section{Statistical Analyses}

To compare the differences between the switched and continuous groups, a Student's $t$-test was used for continuous parametric variables. The Kruskal-Wallis test was used for continuous non-parametric variables, while the chi-square test was used for categorical variables. The time to switch biologics along with type 2 inflammatory biomarkers, pulmonary function indices $\left(\mathrm{FEV}_{1}, \%\right.$ predicted, $\left.\mathrm{FEV}_{1} / \mathrm{FVC}\right)$, JESREC score, and the presence of AERD were assessed using the Kaplan-Meier method. The hazard ratio for switching biologics was derived from the multivariate Cox proportional hazards model. The models were adjusted for age, sex, AERD, $\mathrm{FEV}_{1}, \%$ predicted $<80 \%$, and JESREC scores $\geq 11$. Statistical significance was set at $\mathrm{p}<0.05$. Statistical analyses were performed using the JMP software (SAS Institute Inc., Cary, NC, USA).

\section{Results}

\section{Comparisons Between the Switched Group and the Continuous Group}

Of the 45 patients in whom biologics were introduced, three who discontinued omalizumab before the launch of mepolizumab were excluded. Thus, 42 patients were included in the analysis. Thirteen (31\%) patients required switching biologics (Table 1). Thirteen patients switched biologics, among whom eight and five patients received mepolizumab and benralizumab as the baseline agents, respectively. The subjects in the switched group were significantly younger than those in the continuous group at baseline. The blood eosinophil counts were significantly higher in the switched group than that in the continuous group, while $\mathrm{FEV}_{1}, \%$ predicted and $\mathrm{FEV}_{1} / \mathrm{FVC}$ were not significantly different between the two groups. Regarding comorbidities, ECRS and AERD were more common in the switched group. The reasons for switching were poor control of asthma in eight patients, ECRS in one patient, EOM in one patient, urticaria in one patient, an adverse event in one patient, and economic reasons in one patient (Figure 1). Eight patients with asthma switched biologics because of symptoms of "uncontrolled asthma." Some of these patients required administration of additional oral or systemic corticosteroids or unscheduled hospital visits. In terms of frequency, switching biologics was observed once in six patients, twice in five patients, and thrice in two patients (Figure 1). Ultimately, one, two, four, and six patients switched to omalizumab, mepolizumab, benralizumab, and dupilumab, respectively, at the end of the analysis period (Table 1). Table 2 shows the clinical findings associated with ECRS. The presence of nasal polyps and olfactory cleft lesions were significantly more prevalent in the switched group than in the continuous group.

\section{Factors Associated with Time to Switch Biologics}

The average number of days to switch biologics was 975 (363 days-1338 days), which was significantly shorter in the group with JESREC scores of $\geq 11$ than in the group with JESREC scores of $<11$ (1053 days vs 1480 days) $(\mathrm{P}<0.05)$ (Figure 2A). Patients with AERD also had a significantly shorter time to switch biologics than patients without AERD (750 days vs 1346 days) $(\mathrm{P}<0.05$ ) (Figure $2 \mathrm{~B}$ ). The multivariate Cox proportional hazards model revealed that a JESREC score of $\geq 11$ and younger age were independently associated with the time to switch biologics, but not the presence of AERD and $\mathrm{FEV}_{1}, \%$ predicted (Table 3). 
Table I Demographics of Patients with Asthma in the Continuous and Switched Groups

\begin{tabular}{|c|c|c|c|}
\hline Characteristics & Switched Group & Continuous Group & P-value \\
\hline Number of subjects & 13 & 29 & \\
\hline $\mathrm{Age}^{\dagger *}$, years & $51.0(37.0-65.5)$ & $64.0(50.5-71.0)$ & 0.039 \\
\hline Age at onset of asthma*, years & $32.6(15.3)$ & $43.3(16.3)$ & 0.050 \\
\hline Female sex & $8(61.5)$ & $22(75.9)$ & 0.349 \\
\hline Total lgE, IU/mL & $265(130-364)$ & $171.5(97.9-370)$ & 0.435 \\
\hline Blood eosinophil count* (cells $/ \mu \mathrm{L}$ ) & $706.0(130.5-1049.5)$ & $298.0(|34.5-5| 7.5)$ & 0.047 \\
\hline Atopy & $6(46.2)$ & $13(44.8)$ & 0.331 \\
\hline \multicolumn{4}{|l|}{ Pulmonary function tests } \\
\hline $\mathrm{FEV}_{1}, \mathrm{~L}$ & $2.00(1.69-2.52)$ & $1.58(1.02-2.07)$ & 0.333 \\
\hline $\mathrm{FEV}_{\mathrm{I}}, \%$ predicted $(\%)$ & $76.0 \pm 6.1$ & $76.3 \pm 4.5$ & 0.644 \\
\hline $\mathrm{FEV}_{\mathrm{l}} / \mathrm{FVC}<70 \%$ & $8(61.5)$ & $21(72.4)$ & 0.485 \\
\hline $\mathrm{FeNO}(\mathrm{ppb})$ & $62.5(14.8-106.8)$ & $44.0(24.0-66.0)$ & 0.173 \\
\hline \multicolumn{4}{|l|}{ Treatment } \\
\hline ICS $\operatorname{dose}^{\ddagger}(\mu \mathrm{g})$ & $1800(1500-2800)$ & $1600(1500-2320)$ & 0.193 \\
\hline Maintenance OCS use & $5(38.5)$ & $14(63.6)$ & 0.149 \\
\hline LAMA use & $13(44.8)$ & $4(25.0)$ & 0.189 \\
\hline LTRA use & $12(75.0)$ & $22(75.9)$ & 0.949 \\
\hline Biologics at baseline* & $0 / 8 / 5 / 0$ & $10 / 3 / 11 / 5$ & 0.001 \\
\hline \multicolumn{4}{|l|}{ (Oma/Mep/Ben/Dup) } \\
\hline Biologics after switching & $1 / 2 / 4 / 6$ & $10 / 3 / 11 / 5$ & 0.115 \\
\hline \multicolumn{4}{|l|}{ (Oma/Mep/Ben/Dup) } \\
\hline \multicolumn{4}{|l|}{ Comorbidities } \\
\hline$A R$ & $4(26.7)$ & $6(20.7)$ & 0.765 \\
\hline JESREC II or higher ${ }^{\S * *}$ & $8(61.5)$ & $8(27.6)$ & 0.036 \\
\hline EOM & $4(25.0)$ & II (37.9) & 0.336 \\
\hline AERD* & $6(46.2)$ & $3(10.3)$ & 0.011 \\
\hline
\end{tabular}

Notes: Data are shown as the mean \pm standard deviation, median (interquartile range), or number (\%). P-values were obtained using the chi-square test, Student's $t$-test or the Kruskal-Wallis test, as appropriate. ${ }^{*} \mathrm{P}<0.05^{\dagger}$ Age at the time of introduction of biologics. ${ }^{\ddagger}$ Equivalent to budesonide dose. ${ }^{\S} \mathrm{ECRS}$ was defined as JESREC II or higher.

Abbreviations: AERD, aspirin-exacerbated respiratory disease; AR, allergic rhinitis; ben, benralizumab; dup, dupilumab; ECRS, eosinophilic chronic rhinosinusitis; EOM, eosinophilic otitis media; FeNO, fractional exhaled nitric oxide; $\mathrm{FEV}_{\mathrm{l}}$, forced expiratory volume in I s; FVC, forced vital capacity; Ppb, parts per billion; ICS, inhaled corticosteroids; JESREC, Japanese Epidemiological Survey of Refractory Eosinophilic Chronic Rhinosinusitis; LAMA, longacting muscarinic antagonist; LTRA, Leukotriene Receptor Antagonist; mep, mepolizumab; oma, omalizumab.

\section{Discussion}

The findings of this retrospective study suggest that patients with asthma who required switching biologics were treated with mepolizumab and benralizumab and characterized by the presence of ECRS (defined by JESREC scores of $\geq 11$ ) or AERD, younger age, and higher eosinophil counts. Moreover, the time to switch biologics was significantly shorter in asthma patients with ECRS or AERD compared to their counterparts. Multivariate analysis revealed that JESREC scores of $\geq 11$ and younger age were independently associated factors for switching biologics. Thus, a multidimensional approach when using biologics, including the evaluation of comorbidities, would improve the management of severe asthma, especially with anti-IL-5 agents.

In the present study, all biologics were initiated for poor asthma control by pulmonary physicians. The primary endpoints in most of the previous studies on biologics have solely focused on asthma-related outcomes, such as exacerbations, asthma symptoms, pulmonary function, and the reduction in the OCS dosage. However, it should be emphasized that patients with severe asthma often have comorbidities such as ECRS, AR, GERD, and AD. Interestingly, this study showed that ECRS underlies the switching of biologics, although the worsening of ECRS was an immediate reason for switching biologics in only one patient. This result may highlight the confounding effect of ECRS in treatment with biologics. 


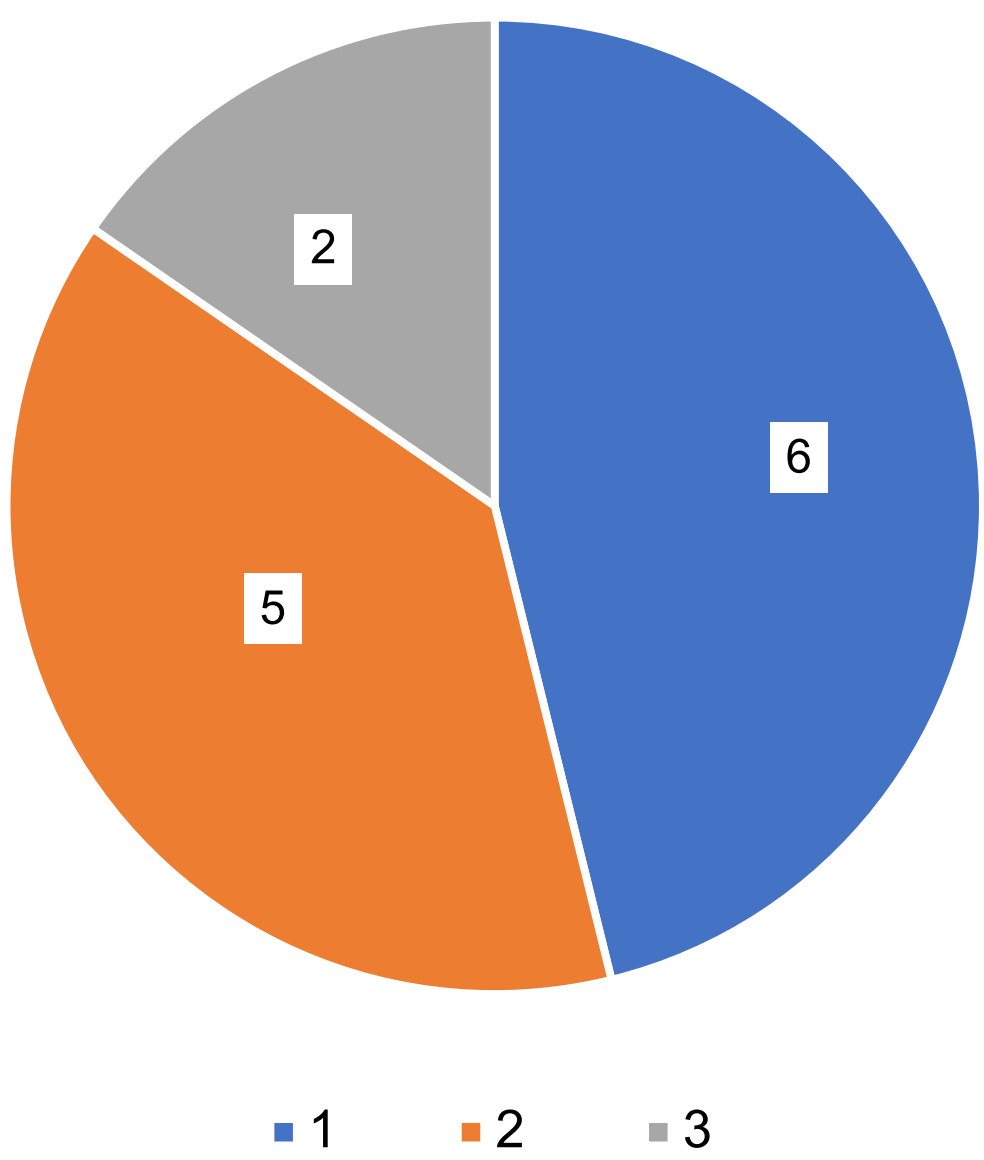

Figure I Frequency of switching biologics in the switched group. Switching biologics was observed once in six patients, twice in five patients, and three times in two patients.

Crosstalk between ECRS and asthma has been suggested, which highlights the need for the assessment of ECRS in severe asthma. ${ }^{18}$ Eger et al have shown that sinonasal comorbidities are a feature in partial responders $(69 \%$ of the total subjects) with residual disease manifestation, which resulted in switching biologics. ${ }^{10}$ Akaba et al have also reported that CRSwNP was more prevalent in those who switched than in those who continued first-line biologics. ${ }^{19}$ In this study, we identified ECRS, a severe phenotype of CRSwNP, as an associated factor for switching biologics. The JESREC score is a criterion based on the findings of sinus CT and blood tests, which is often available in clinical practice. ${ }^{15,20}$ Notably, a high hazard ratio of switching biologics with a JESREC score $\geq 11$ was observed, independent of age, sex, AERD, and $\mathrm{FEV}_{1}, \%$ predicted $<80 \%$. Thus, our results have confirmed and broadened previous findings. Meanwhile, younger age was an independent risk factor affecting the time to switch. Patients with asthma were significantly younger in the switched group than those in the continuous group (51.0 [37.0-65.5] and 64.0 [50.5-71.0] years old, respectively) (Table 1). Non-responders to anti-IL-5 antibody drugs were significantly younger at baseline than responders, ${ }^{21}$ which may be concordant to poor control of asthma at younger ages, which resulted in switches in the present study. Thus, it might be possible that younger patients require more switches to find the optimal biologics. This should be validated in future studies.

Reports have suggested that the responses of the upper and lower airways to biologics can vary in asthmatics. ${ }^{11,12}$ In a previous case report, asthma was well controlled with benralizumab, while comorbid ECRS responded better to mepolizumab than to benralizumab. ${ }^{11}$ In contrast, the coexistence of nasal polyps is predictive of a better response to benralizumab. ${ }^{21}$ Benralizumab improves QoL of patients with severe eosinophilic asthma, especially in those with concomitant nasal polyps. ${ }^{22} \mathrm{~A}$ switch could be considered when the upper airway disease is not controlled by biologics in 
Table 2 Factors Related to Eosinophilic Chronic Rhinosinusitis in the Continuous and Switched Groups

\begin{tabular}{|l|l|l|l|}
\hline Characteristics & Switched Group & Continuous Group & P-value \\
\hline Number of subjects & 13 & 29 & \\
JESREC score*, points & $11.0(7.5-17.0)$ & $8.0(4.0-10.5)$ & 0.030 \\
Olfactory cleft lesion* & $7(54)$ & $4(14)$ & 0.008 \\
Nasal polyp* & $6(55)$ & $5(20)$ & 0.042 \\
Affected side: both sides & $8(73)$ & $10(42)$ & 0.083 \\
Ethmoid-dominant inflammation & $7(64)$ & $7(29)$ & 0.054 \\
Severe ECRS & $5(7 I)$ & $4(57)$ & 0.833 \\
CT grading score (Lund-Mackay), point & $11(0-20.0)$ & $1(0-6.8)$ & 0.150 \\
\hline
\end{tabular}

Notes: Data are shown as the median (interquartile range), or number (\%). P-values were obtained using the chi-square test or the Kruskal-Wallis test, as appropriate. $* \mathrm{P}<0.05$.

Abbreviations: ECRS, eosinophilic chronic rhinosinusitis; JESREC, Japanese Epidemiological Survey of Refractory Eosinophilic Chronic Rhinosinusitis; CT, computed tomography.

patients with the coexistent asthma and upper airway disease. However, no predictors for responses have been elucidated. ${ }^{23}$ Conclusively, the efficacy in biologics should be evaluated in all organs in individuals.

The prevalence of AERD has been estimated to be $5.5-12.4 \%$ in asthma and as high as $14.9 \%$ in severe asthma. ${ }^{24}$ Furthermore, nearly $9.7 \%$ of asthmatics with chronic rhinosinusitis with nasal polyps have AERD. ${ }^{24}$ AERD is one of the factors contributing to a deterioration in clinical outcomes and refractory asthma Omalizumab has been reported as a candidate for the treatment of AERD. ${ }^{25}$ In this study, AERD was more common in the switched group, which may be explained by the high rate of coexistent AERD and ECRS. This is reflected by the finding that ECRS was an independently associated factor for the time to switching biologics and not AERD.

Higher BMI, more respiratory symptoms, fewer nasal polyps, lower $\mathrm{FEV}_{1}$, and higher doses of OCS have been suggested as the characteristics of non-responders to biologics. ${ }^{9}$ Previous reports on anti-IL-5 biologics showed that $\mathrm{FEV}_{1}, \%$ predicted of $>80 \%$ and less than 10 years of duration of asthma were associated factors in super responders; however, there were no independent clinically distinctive factors in non-responders. ${ }^{11}$ None of the abovementioned characteristics of non-responders correlated with switching biologics in the present study, which may imply that asthma control is not the sole reason for the discontinuation of biologics. Notably, there were no differences in the maintenance drugs at baseline between the switched and continuous groups besides biologics, such as the doses of ICS and the use of
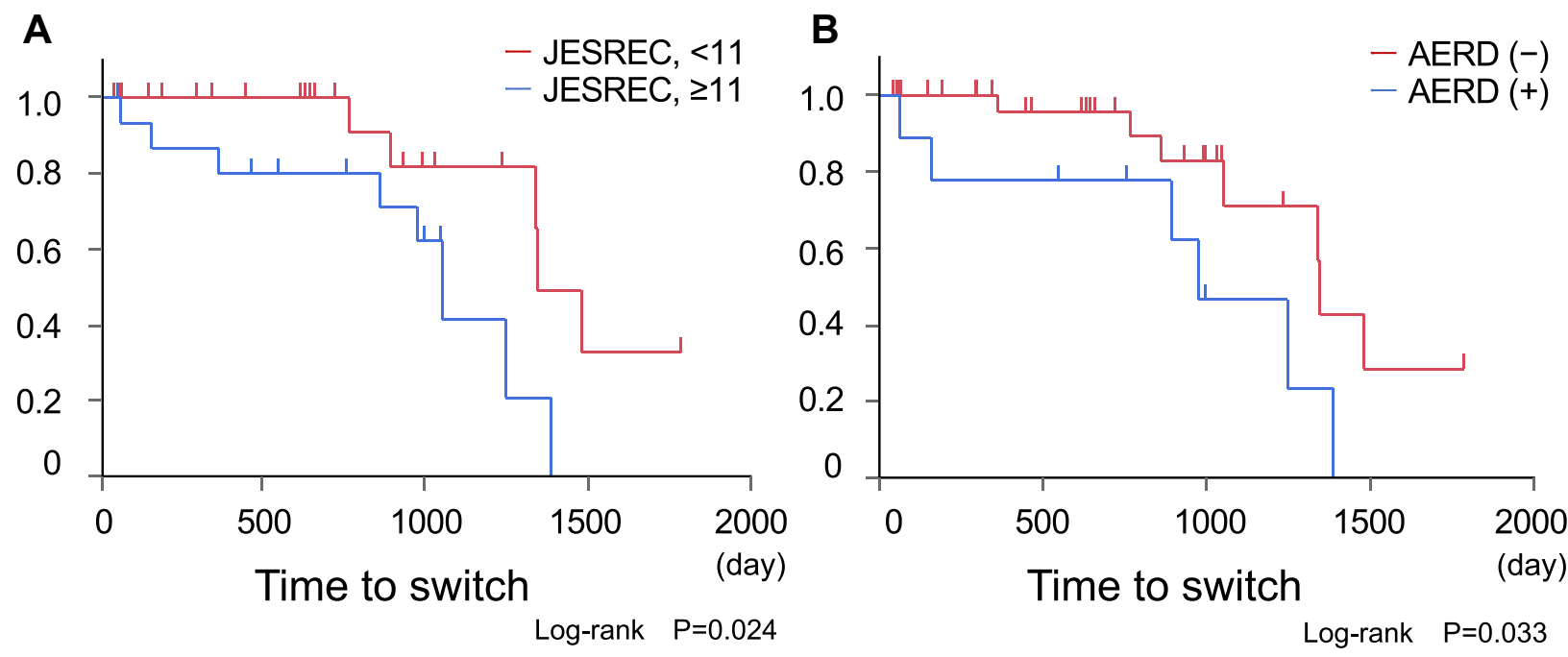

Figure 2 Kaplan-Meier curves for time to switch the biologics. (A) The subgroup analysis on JESREC score. The time to switch biologics was significantly shorter in the subgroup with the JESREC score of II or higher than that with the JESREC score $<$ II $(P<0.05)$. (B) The subgroup analysis on AERD. The time to switch biologics was significantly shorter in the subgroup with AERD than that without AERD $(P<0.05)$. 
Table 3 Associated Factors with Time to Switch Biologics

\begin{tabular}{|l|l|l|}
\hline Variable & Hazard Ratio $\mathbf{( 9 5 \% ~ C l )}$ & P-value \\
\hline Age $^{\dagger *}$ & $0.91(0.84-0.96)$ & 0.004 \\
Female sex & $0.20(0.01-2.67)$ & 0.224 \\
FEV $_{1}, \%$ predicted & $1.03(0.46-1.08)$ & 0.137 \\
JESREC, $\geq 1 I^{*}$ & $20.8(1.78-240.9)$ & 0.015 \\
AERD & $0.41(0.06-2.8)$ & 0.362 \\
\hline
\end{tabular}

Notes: $* \mathrm{P}<0.05 .{ }^{\dagger}$ Age at time of biologics introduction.

Abbreviations: AERD, aspirin-exacerbated respiratory disease; $\mathrm{FEV}_{1}$, forced expiratory volume in I

s; JESREC, Japanese Epidemiological Survey of Refractory Eosinophilic Chronic Rhinosinusitis.

long-acting muscarinic antagonists, leukotriene receptor antagonists, and OCS (Table 1). Moreover, no significant modification was found in those drugs during the observed periods, which strengthens the results of this study.

This study has several limitations. First, given the nature of a retrospective study, there were no clear criteria for stopping or switching the therapy. Thus, the decision of discontinuing or switching biologics depended on the attending physician. Nevertheless, considering the current state in which no criteria have been established for a switch, findings obtained in real-world settings are of value. Second, the sample size of this study was relatively small. This may considerably affect the results, reflected by the wide confidence interval. Third, three patients had been treated by other biologics before their entry to the present study, which might affect the findings. Lastly, the indications and clinical availabilities of the four biologics varied in Japan, which may have affected the results of this study. Dupilumab was indicated for ECRS and AD, and omalizumab was indicated for seasonal AR and idiopathic chronic urticaria besides asthma, while mepolizumab and benralizumab were indicated for asthma only. Omalizumab has been available for asthma treatment since March 2009, mepolizumab since June 2016, benralizumab since April 2018, and dupilumab since March 2019. Dupilumab became available for the treatment of ECRS in March 2020. Thus, future studies should judge the effects of the biases and examine the results of these studies.

\section{Conclusion}

ECRS assessed by a JESREC score of $\geq 11$ was associated with switching biologics. This highlights the significance of assessing comorbidities in the treatment of asthma with biologics. The selection and switching of biologics should be considered holistically and not just based on the organ system.

\section{Abbreviations}

ACT, Asthma Control Test; AD, atopic dermatitis; AERD, aspirin-exacerbated respiratory disease; AR, allergic rhinitis; CRSwNP, chronic rhinosinusitis with nasal polyps; CT, computed tomography; ECRS, eosinophilic chronic rhinosinusitis; EOM, eosinophilic otitis media; FeNO, fractional exhaled nitric oxide; $\mathrm{FEV}_{1}$, forced expiratory volume in 1 second; FVC, forced vital capacity; GERD, gastroesophageal reflux disease; ICS, Inhaled corticosteroids; JESREC, Japanese Epidemiological Survey of Refractory Eosinophilic Chronic Rhinosinusitis; LABA, long-acting beta2agonist; OCS, oral corticosteroids.

\section{Ethics Approval and Informed Consent}

The study was approved by the Ethical Review Board for Life Science and Medical Research, Hokkaido University Hospital [approval number: 021-0080]. Informed consent was obtained in the form of an opt-out on the website.

\section{Acknowledgments}

We thank Dr. Munehiro Matsumoto, Dr. Hiroki Kimura, Dr. Sho Nakakubo, Dr. Akira Oguma, Dr. Keisuke Kamada, Dr. Yuki Abe, Dr. Nozomu Takei and Dr. Yu Yamashita, for the clinical assistance for this study. 


\section{Author Contributions}

MMS, KS, and Masaru Suzuki designed the study and drafted the manuscript. HK, Masanobu Suzuki and SK contributed to data collection. Masanobu Suzuki, YN and AH performed otolaryngological examination. MMS performed the statistical analysis and MMS and YI performed interpretation of the result. All authors contributed to data analysis, drafting or revising the article, agreed on the journal to which the article will be submitted, gave final approval of the version to be published, and agree to be accountable for all aspects of the work.

\section{Disclosure}

Masaru Suzuki reports Lecture fee from GlaxoSmithKline, Novartis, and AstraZeneca outside the current work. YI reports consulting fees from JAPAN TOBACCO INC., Kowa Company, Limited, Nipro Corporation, ONO PHARMACEUTICAL CO., LTD and payment for speaker from Novartis Japan and participation on a Data safety monitoring Board or Advisory Board from Janssen Pharmaceutical, K.K. outside the current work. SK received grants from AstraZeneca, Kyorin Pharmaceutical, Nippon Boehringer Ingelheim, Novartis and payment for speaker from Astra Zeneca and Nippon Boehringer Ingelheim. The rest of the authors declare that they have no relevant conflicts of interest. The above-mentioned companies or organizations has not been involved in the concept or analysis of the present work.

\section{References}

1. Pelaia C, Crimi C, Vatrella A, Tinello C, Terracciano R, Pelaia G. Molecular targets for biological therapies of severe asthma. Front Immunol. 2020;11:603312. doi:10.3389/fimmu.2020.603312

2. Global Initiative for Asthma. Global Strategy for Asthma Management and Prevention (2021 update). Available from: https://ginasthma.org/ginareports/ Accessed October 27, 2021.

3. Chung KF, Wenzel SE, Brozek JL, et al. International ERS/ATS guidelines on definition, evaluation and treatment of severe asthma. Eur Respir J. 2014;43(2):343-373. doi:10.1183/09031936.00202013

4. Thomas M, Kay S, Pike J, et al. The Asthma Control Test (ACT) as a predictor of GINA guideline-defined asthma control: analysis of a multinational cross-sectional survey. Prim Care Respir J. 2009;18(1):41-49. doi:10.4104/pcrj.2009.00010

5. Nakamura Y, Tamaoki J, Nagase H, et al. Japanese guidelines for adult asthma 2020. Allergol Int. 2020;69(4):519-548. doi:10.1016/j. alit.2020.08.001

6. Chan R, RuiWen Kuo C, Lipworth B. Pragmatic clinical perspective on biologics for severe refractory Type 2 asthma. J Allergy Clin Immunol Pract. 2020;8(10):3363-3370. doi:10.1016/j.jaip.2020.06.048

7. Bousquet J, Brusselle G, Buhl R, et al. Care pathways for the selection of a biologic in severe asthma. Eur Respir J. 2017;50:6. doi:10.1183/ 13993003.01782-2017

8. Kroes JA, Zielhuis SW, van Roon EN, Ten Brinke A. Prediction of response to biological treatment with monoclonal antibodies in severe asthma. Biochem Pharmacol. 2020;179:113978. doi:10.1016/j.bcp.2020.113978

9. Kavanagh JE, d'Ancona G, Elstad M, et al. Real-world effectiveness and the characteristics of a "super-responder" to mepolizumab in severe eosinophilic asthma. Chest. 2020;158(2):491-500. doi:10.1016/j.chest.2020.03.042

10. Eger K, Kroes JA, Ten Brinke A, Bel EH. Long-term therapy response to anti-il-5 biologics in severe asthma-a real-life evaluation. $J$ Allergy Clin Immunol Pract. 2021;9(3):1194-1200. doi:10.1016/j.jaip.2020.10.010

11. Matsumoto-Sasaki M, Shimizu K, Suzuki M, Nakamaru Y, Konno S. [A case of severe asthma switched to mepolizumab due to late-occurring unresponsiveness to benralizumab]. Arerugi. 2020;69(8):678-682. Japanese. doi:10.15036/arerugi.69.678

12. Matsumoto-Sasaki M, Shimizu K, Suzuki M, Nakamaru Y, Konno S. [A case of severe asthma who required a switch of four biologics: differential responses on upper and lower airways]. Arerugi. 2021;70(4):315-320. Japanese. doi:10.15036/arerugi.70.315

13. Dweik RA, Boggs PB, Erzurum SC, et al. An official ATS clinical practice guideline: interpretation of exhaled nitric oxide levels (FENO) for clinical applications. Am J Respir Crit Care Med. 2011;184(5):602-615. doi:10.1164/rccm.9120-11ST

14. Tojo N, Suga H, Kambe M. [Lung function testing -the Official Guideline of the Japanese Respiratory Society]. Rinsho Byori. $2005 ; 53(1): 77-81$. Japanese.

15. Tokunaga T, Sakashita M, Haruna T, et al. Novel scoring system and algorithm for classifying chronic rhinosinusitis: the JESREC Study. Allergy. 2015;70(8):995-1003. doi:10.1111/all.12644

16. Laidlaw TM, Boyce JA. Aspirin-exacerbated respiratory disease-new prime suspects. N Engl J Med. 2016;374(5):484-488. doi:10.1056/ NEJMcibr1514013

17. Iino Y, Tomioka-Matsutani S, Matsubara A, Nakagawa T, Nonaka M. Diagnostic criteria of eosinophilic otitis media, a newly recognized middle ear disease. Auris Nasus Larynx. 2011;38(4):456-461. doi:10.1016/j.anl.2010.11.016

18. Tajiri T, Fujita S, Sokai A, et al. Effect of endoscopic sinus surgery for chronic rhinosinusitis on the state of coexisting asthma. Allergol Int. 2020;69 (2):279-280. doi:10.1016/j.alit.2019.12.001

19. Akaba T, Kondo M, Muramatsu S, et al. Clinical characteristics and treatment outcomes of severe asthma patients with a history of multiple biologic drugs use. Asian Pac J Allergy Immunol. 2021. doi:10.12932/ap-170221-1070

20. Fujieda S, Imoto Y, Kato Y, et al. Eosinophilic chronic rhinosinusitis. Allergol Int. 2019;68(4):403-412. doi:10.1016/j.alit.2019.07.002

21. Bleecker ER, Wechsler ME, FitzGerald JM, et al. Baseline patient factors impact on the clinical efficacy of benralizumab for severe asthma. Eur Respir J. 2018;52:4. doi:10.1183/13993003.00936-2018 
22. Scioscia G, Carpagnano GE, Quarato CMI, et al. Effectiveness of benralizumab in improving the quality of life of severe eosinophilic asthmatic patients: our real-life experience. Front Pharmacol. 2021;12:631660. doi:10.3389/fphar.2021.631660

23. Papaioannou AI, Fouka E, Papakosta D, Papiris S, Loukides S. Switching between biologics in severe asthma patients. When the first choice is not proven to be the best. Clin Exp Allergy. 2021;51(2):221-227. doi:10.1111/cea.13809

24. Rajan JP, Wineinger NE, Stevenson DD, White AA. Prevalence of aspirin-exacerbated respiratory disease among asthmatic patients: a meta-analysis of the literature. J Allergy Clin Immunol. 2015;135(3):676-81.e1. doi:10.1016/j.jaci.2014.08.020

25. Hayashi H, Fukutomi Y, Mitsui C, et al. Omalizumab for aspirin hypersensitivity and leukotriene overproduction in aspirin-exacerbated respiratory disease. A randomized controlled trial. Am J Respir Crit Care Med. 2020;201(12):1488-1498. doi:10.1164/rccm.201906-1215OC

\section{Publish your work in this journal}

The Journal of Asthma and Allergy is an international, peer-reviewed open-access journal publishing original research, reports, editorials and commentaries on the following topics: Asthma; Pulmonary physiology; Asthma related clinical health; Clinical immunology and the immunological basis of disease; Pharmacological interventions and new therapies. The manuscript management system is completely online and includes a very quick and fair peer-review system, which is all easy to use. Visit http://www.dovepress.com/testimonials.php to read real quotes from published authors.

Submit your manuscript here: https://www.dovepress.com/journal-of-asthma-and-allergy-journal 